\title{
User-guided line abstraction using coherence and structure analysis
}

\author{
Hui-Chi Tsai ${ }^{1,2}$, Ya-Hsuan Lee ${ }^{1}$, Ruen-Rone Lee $^{2}(\bowtie)$, and Hung-Kuo Chu ${ }^{1}(\varangle)$ \\ (c) The Author(s) 2016. This article is published with open access at Springerlink.com
}

Abstract Line drawing is a style of image abstraction where the perceptual content of the image is conveyed using distinct straight or curved lines. However, extracting semantically salient lines is not trivial and mastered only by skilled artists. While many parametric filters have successfully extracted accurate and coherent lines, their results are sensitive to parameter choice and easily lead to either an excessive or insufficient number of lines. In this work, we present an interactive system to generate concise line abstractions of arbitrary images via a few user specified strokes. Specifically, the user simply has to provide a few intuitive strokes on the input images, including tracing roughly along edges and scribbling on the region of interest, through a sketching interface. The system then automatically extracts lines that are long, coherent and share similar textural structures to form a corresponding highly detailed line drawing. We have tested our system with a wide variety of images. Our experimental results show that our system outperforms state-of-the-art techniques in terms of quality and efficiency.

Keywords line abstraction; interactive drawing; coherence strokes; structure strokes; stroke matching

1 Department of Computer Science, "National Tsing Hua University", No. 101, Section 2, Kuang-Fu Road, Hsinchu, Taiwan 30013, China. E-mail: H.-C. Tsai, beck394@itri.org.tw; Y.-H. Lee, louiselee602@gmail.com; H.-K. Chu, hkchu@cs.nthu.edu.tw ( $₫)$.

2 Information and Communications Research Laboratories, Industrial Technology Research Institute, No. 195, Section 4, Chung Hsing Road, Chutung, Hsinchu, Taiwan 31040, China. E-mail: rrlee@itri.org.tw (四).

Manuscript received: 2016-09-09; accepted: 2016-12-21

\section{Introduction}

Line drawing is a style of image abstraction in which a distinct and concise set of line strokes is used to depict the shapes of objects in a scene. Such concise image abstraction plays a fundamental element in many artistic stylizations where the artists delicately draw long coherent lines along semantically salient features in an image to give a first impression of their artworks. Beyond artistic drawings, good line abstraction also provides valuable priors for advanced image processing and scene understanding tasks that demand precise edge detection.

However, generating semantically meaningful line abstractions is not a trivial task; it is currently approached in two very different ways. One way, mostly appreciated by artists, is to utilize various commercial painting tools (e.g., Paint, Photoshop) to precisely trace the salient features by hand. Although this offers the artists full control over the final results, the process is tedious, time-consuming, and probably error-prone due to fatigue. In the other approach, a huge body of work is dedicated to automatic line abstraction in various contexts, ranging from gradient-based edge detection [1] to artistic abstraction [2]. While such automation largely eliminates the manual effort required and achieves pixel level accuracy, the results are highly sensitive to parameter settings, leading to either excessive or insufficient detail. Overall, we still lack an efficient and effective technique to extract concise yet semantically meaningful line abstractions from images.

In this work, we present a novel line abstraction algorithm to extract prominent line strokes from a highly detailed line drawing under the supervision of a few user specified strokes. The key insight is 
to leverage both the cognitive ability of humans and the computational power of machines to accomplish the line abstraction task. To minimize the fatigue, the user simply has to scribble roughly along the long image features (e.g., contours of objects) or on a region with similar texture patterns via a sketching interface. The system then automatically performs the accuracy-demanding and computationally expensive tasks of extracting a concise yet semantically meaningful line abstraction from a highly detailed one. Specifically, the system first classifies the user strokes into coherence and structure strokes, based on which the long coherent lines and line segments that share similar texture patterns in the input image are extracted, respectively. Figure 1 shows a typical example generated by our system using only a few handdrawn strokes. We have tested our system on a variety of images across different users. Experimental results show that our system can generate superior or comparable line abstractions given the same input user strokes in comparison to previous state-of-theart methods, and provides a significant performance boost over hand drawing when a target complexity of abstraction is requested.

In summary, our main contributions include:

- An easy-to-use sketching system that facilitates the creation of concise, semantic line abstractions using very simple and intuitive user strokes.

- A novel line matching algorithm for extracting long coherent lines and line segments with similar image domain structure using coherence and structure strokes respectively, that are automatically derived from the user strokes.

\section{Related work}

Parametric image filtering. Parametric image filters such as the Canny edge detector [1] and the
difference-of-Gaussians filter [2-4] are widely used in image abstraction for generating line drawing images. However, the quality of output may vary significantly when adjusting the associated control parameters, leading to either excessive or insufficient details. Another well-known contour detector, global probability of boundary $(\mathrm{gPb})[5,6]$ combines both local and global image features, and requires a single threshold parameter to control the number of detected edges. Nevertheless, it remains difficult to find a universally applicable setting that produces satisfactory results for different input images. Rather than struggling to optimize parameters, our work aims to utilize these well-defined filters to generate over-detailed line drawing images, from which a concise set of semantic lines is then extracted via the user specified strokes.

Sketch-based refinement. Limpaecher et al. [7] introduced a method to correct user input strokes by a consensus model collected from a crowdsourced drawing database. $\mathrm{Su}$ et al. [8] presented the EZ-Sketching system that snaps user strokes to nearby edges using a novel three-level optimization. These systems also resemble those that snap the cursor or strokes to some specific image features such as image snapping [9] and lazy snapping [10]. Other interactive sketching systems such as ShadowDraw [11], drawing assistant [12], and iCanDraw [13] targeted providing a tutor-like drawing system for novice users. In contrast to previous works that intend to correct or guide user strokes, our system aim to use user stokes as guidance to effectively extract prominent lines that match the user's intentions from a detailed line drawing image.

Stylized line drawing. RealBrush [14] used scanned images of real natural media to synthesize the texture appearance of user strokes. A portrait sketching system by Berger et al. [15] is capable

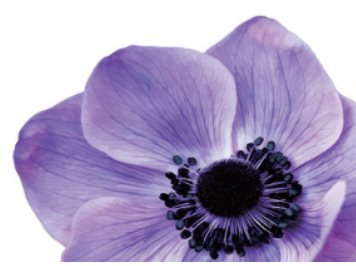

(a)

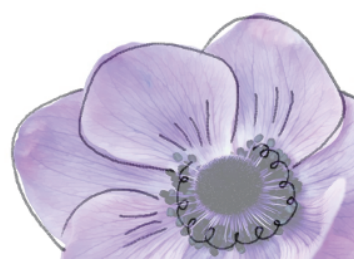

(b)

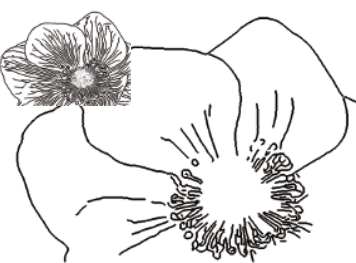

(c)

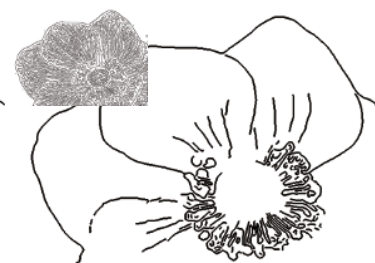

(d)

Fig. 1 Given an input image (a) along with a few scribbles by the user (b), our system automatically extracts a concise line abstraction with coherence and structure lines depicting the edges of the petals and the shapes of the pistils (c, d). Note that our system can adaptively produce highly detailed line drawings using different image filters (see insets). 
of synthesizing a sketch portrait from a photograph that mimics a particular artistic style. Both systems are data-driven and achieve impressive results by analyzing the relationship between input strokes and the collected line abstraction database. Our system can contribute to this line of work by serving as an efficient tool for generating line abstractions with various styles.

Our work is closely related to the work by Yang et al. [16] who also tried to extract semantic gradient edges based on input user strokes. Their system first clusters edge points into edgelets and constructs a graph that encodes the spatial relations between the edges near the user strokes. An energy minimization framework is then used to select the semantic edges that conform to the shapes of the user strokes. However, their line matching algorithm may produce artifacts such as disconnected edges even if the input strokes are coherent. Moreover, lacking support for structure analysis in the texture domain, their system requires users to provide strokes at different scales in order to extract the corresponding gradient edges.

\section{Overview}

An overview of our system is provided in Fig. 2. Given an input image, our system starts by the user providing rough scribbles on the regions of interest (see Fig. 2(b)) to guide line abstraction. In addition to the user strokes, our system also takes as input a detailed line drawing of the input image (see Fig. 2(c)), which provides a reference dataset of line segments used in the subsequent matching algorithm. Such a detailed line drawing can be obtained by using any suitable well-known image filter, such as the Canny edge detector [1], fDoG [2], $\mathrm{gPb}[5,6]$, etc. Then our system runs in two stages.

Stroke classification. The user has to provide only two kinds of simple and intuitive strokes: (i) roughly tracing long image features (e.g., outlines, edges), and (ii) scribbling on the regions using zigzag or circular strokes. We refer to the former type of strokes as coherence strokes. These are simple lines that are nearly straight, and are usually used to depict the main shape of objects (see Fig. 2(d)). The other stroke type, structure strokes, are mainly used to indicate regions of interest that contain repeated texture patterns, which are otherwise tedious to trace by hand (see Fig. 2(e)). Since these two types of strokes represent different intentions of the user, our system employs a gesture recognition technique [17] to classify the user strokes.

Line matching. We formulate line abstraction as a line matching problem, the aim being to extract lines from the reference dataset that match the user specified coherence and structure strokes (if any). Specifically, for each coherence stroke, the system computes the best matching coherence lines (see Fig. 2(f)) using metrics favoring candidate lines that are smooth and in agreement with the user stroke in terms of orientation and overall length (see

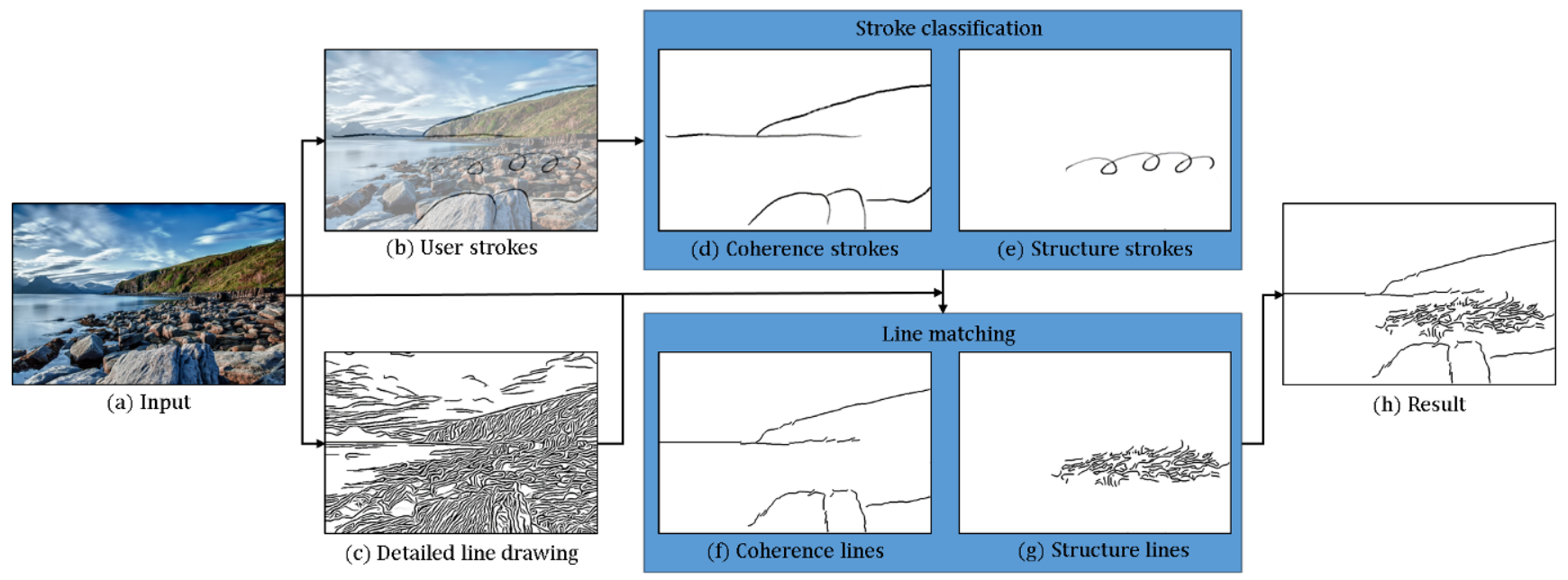

Fig. 2 Overview. Given an input image (a), the system lets the user provide a few simple, intuitive strokes (b) and generates a reference dataset of line segments from a detailed line drawing image (c). Next, the system classifies the user strokes into coherence strokes (d) and structure strokes (e). A novel line matching algorithm is then employed to match the line segments of (c) to the input coherence and structure strokes. The best matching coherence lines (f) and structure lines (g) are combined to form the final line abstraction (h). 
Section 4.1). For each structure stroke, the system first analyzes a representative feature descriptor based on texture patches of the input image by sampling along the stroke using a local window. Then the corresponding structure lines are those lines of the reference dataset that are close to the user stroke in feature space (see Fig. 2(g) and Section 4.3). The final result is obtained by combining these two types of extracted lines.

\section{Algorithm}

Preprocessing. The system starts by preprocessing the input detailed line drawing image to obtain a dataset of atomic line segments for the subsequent matching algorithm. This is done by splitting long continuous lines into small line segments according to both length and curvature constraints. Assuming a line comprises a set of $t$ consecutive pixels $\left\{p_{1}, \cdots, p_{t}\right\}$, we measure the curvature of $p_{i}$ using the angle $\theta$ between two vectors, $\overrightarrow{p_{i}, p_{i-2}}$ and $\overrightarrow{p_{i}, p_{i+2}}$. If $\theta$ is less than $135^{\circ}$ or the length of the line exceeds a threshold of 20 pixels, we subdivide the line into two line segments. The splitting process is iterated until no more line segments violate the length and curvature requirements.

We define a region of interest (ROI) for each user stroke to speed up the process, by constraining candidate matching line segments to those intersecting the ROI. The ROI is defined as the region swept by a disk aligned with and moving along the user stroke. We use an empirical setting of 15 pixels as the default disk radius to generate all results presented in the paper.

\subsection{Coherence line matching}

Coherence strokes correspond to the user's intention to trace along the contours of an object to depict its overall shape. Therefore, the goal in this step is to extract line segments to form a long coherent line that matches each user stroke in terms of length and orientation. The details of algorithm are as below.

Graph construction. For each input coherence stroke, the system first constructs a directed graph $G=(V, E)$, with vertex set $V=\left\{v_{1}, \cdots, v_{m}\right\}$ containing all candidate line segments covered by the stroke's ROI; we add a directed edge for every pair of distinct vertices. The edges can be further divided into two types according to context. The edge is labeled as a real edge if its two vertices (i.e., line segments) are originally connected in the source line drawing image, otherwise it is labeled as a virtual edge. We defer the discussion of how to determine the direction of each edge until later. An example of such a digraph can be seen in Fig. 3.

Vertex-wise energy term. Assume the coherence stroke is also split into a set of stroke segments, denoted $S=\left\{s_{1}, \cdots, s_{n}\right\}$. For each vertex $v_{i}$, we search among the set $S$ and assign $v_{i}$ to the best matching stroke segment according to an alignment function, formulated as

$$
C_{\text {align }}\left(v_{i}\right)=\min _{s \in S}\left(C_{\text {distance }}\left(v_{i}, s_{j}\right) C_{\text {angle }}\left(v_{i}, s_{j}\right)\right)
$$

The distance cost, $C_{\text {distance, }}$, calculates the average distance between $v_{i}$ and $s_{j}$, and is defined as

$$
C_{\text {distance }}\left(v_{i}, s_{j}\right)=\frac{1}{N} \sum_{p \in s_{j}} \min _{q \in v_{i}}(\|p-q\|)
$$

The orientation cost, $C_{\text {angle }}$, measures how well $v_{i}$ is aligned with $s_{j}$, and is defined as

$$
C_{\text {angle }}\left(v_{i}, s_{j}\right)=1+\alpha \tan \theta
$$

where $\theta$ represents the acute angle between $v_{i}$ and $s_{j}$, and $\alpha$ is a weight which is empirically set to 2 in our experiments.

Edge-wise energy term. Since our purpose is to extract a path in the graph that is coherent with the input stroke, the edge direction should naturally follow the drawing direction of the input stroke. The edge direction is determined by computing the angle between a pair of line segments $\left(v_{i}, v_{j}\right)$ and their best matching stroke segments $\left(s_{i}, s_{j}\right)$. As shown in Fig. 4(b), we calculate the angle, $\theta$, between the two vectors $\overrightarrow{m_{s_{i}} m_{s_{j}}}$ and $\overrightarrow{m_{v_{i}} m_{v_{j}}}$, where $m_{s}$ and $m_{v}$ are the midpoints of $s_{i}$ and $v_{i}$, respectively.

The edge direction is set from $v_{i}$ to $v_{j}$ if $\theta$ is less than $90^{\circ}$, otherwise it is set to the opposite direction.

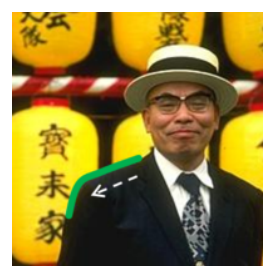

(a)

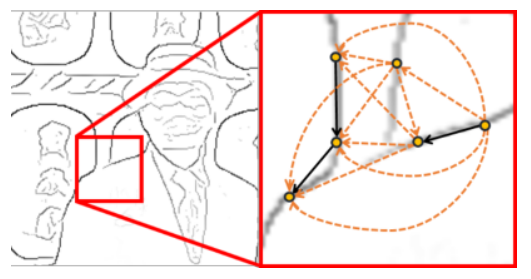

(b)
Fig. 3 Graph construction. (a) A coherence stroke (green) drawn by the user; direction shown by white arrow. (b) (Right) corresponding digraph based on the detailed line drawing (left). Each vertex represents a line segment. Real edges: black arrows. Virtual edges: orange arrows. Directions of edges are consistent with the direction of the input stroke. 


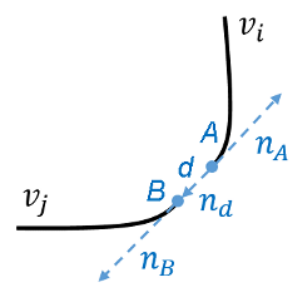

(a)

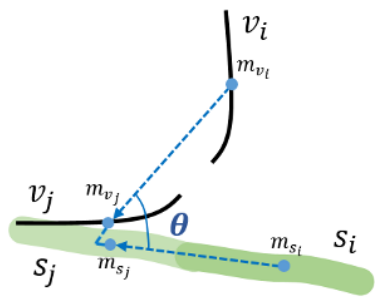

(b)
Fig. 4 Continuity measure for a pair of line segments $v_{i}$ and $v_{j}$. (a) Two line segments with strong continuity in terms of $C_{\text {line }}$. (b) Two line segments with weak continuity in terms of $C_{\text {user }}$. Edge direction is decided by the angle between line segments and the matching stroke segments.

The associated edge weight $W\left(v_{i}, v_{j}\right)$ is determined based on the continuity between each pair of line segments $\left(v_{i}, v_{j}\right)$, defined by

$$
W\left(v_{i}, v_{j}\right)=\frac{C_{\text {line }}+C_{\text {user }}}{2}|d|
$$

The continuity cost, $C_{\text {line }}$, takes into account the geometric feature of the two line segments, and is defined as

$$
C_{\text {line }}=\frac{\left\|n_{A} \times n_{B}\right\|+\left\|n_{A} \times n_{d}\right\|+\left\|n_{B} \times n_{d}\right\|}{3}
$$

where $n_{A}$ and $n_{B}$ are the unit tangent vectors at points $A$ and $B$, which correspond to two points respectively on line segments $v_{i}$ and $v_{j} . d$ is the distance vector from point $A$ to point $B$, and $n_{d}$ is a normalized unit vector along $d$. Figure 4(a) illustrates a case with small $C_{\text {line }}$. For real edges, $C_{\text {line }}$ is set to 1 . Note that Eq. (5) is a slight modification of the discontinuity term introduced in the stage of stroke clustering in Ref. [18].

However, in some cases, two geometrically connected line segments may actually come from two semantically different objects. Take Fig. 3 for instance: although the line on the left hand side of the window is long continuous, it is actually made up of edges from different objects (i.e., the shoulder and the lantern). To handle such cases, we introduce another continuity cost, $C_{\text {user }}$, using the indication from the coherence stroke to determine whether two line segments have strong or weak continuity. This cost function is defined as

$$
C_{\text {user }}=\cos (\theta-\pi / 2)
$$

where $\theta$ is the angle between two line segments and their matching stroke segments. When $\theta$ is large, it means that the user intends weak continuity between two line segments even though they show strong continuity in terms of $C_{\text {line }}$. Figure 4 illustrates a case with small $C_{\text {line }}$ and large $C_{\text {user }}$.
Optimization. Given the directed graph, we apply the Floyd-Warshall algorithm [19] to compute all pairs of shortest paths to find the most coherent path for each pair of vertices. In order to extract the most prominent paths, we define an energy function, $E(p)$, to measure the quality of each path $p$ as follows:

$$
E(p)=a E_{\text {align }}+b E_{\text {length }}+c E_{\text {coherence }}
$$

The alignment energy term, $E_{\text {align }}$, simply averages the alignment cost along the path $p$ and is defined as

$$
E_{\text {align }}=\sum_{v_{i} \in p} C_{\text {align }}\left(v_{i}\right) / N_{p}
$$

where $N_{p}$ is the number of vertices on path $p$. The length energy term, $E_{\text {length }}$, computes the proportion of matched user stroke segments of each path and favors the length of extracted lines to be as close as possible to the coherence stroke. $E_{\text {length }}$ is defined as

$$
E_{\text {length }}=1-N_{\text {matched }} / N_{\text {user }}
$$

where $N_{\text {matched }}$ is the number of matched stroke segments and $N_{\text {user }}$ is the number of stroke segments. For the coherence energy term, $E_{\text {coherence, we simply }}$ average the edge weight along the path $p$ :

$$
E_{\text {coherence }}=\sum_{v_{i}, v_{j} \in p} W\left(v_{i}, v_{j}\right) /\left(N_{p}-1\right)
$$

where $N_{p}-1$ is the number of edges on path $p$. The three energy terms are combined using weighting parameters, $a, b$, and $c$. The path with minimal total energy is selected as the most prominent line that matches the input coherence stroke. Note that we use the empirical values of $a=b=c=1$ as default values to generate all results shown in the paper. Figure 5 shows some results of coherence stroke matching.

\subsection{Temporospatially neighboring strokes}

To distinguish coherence strokes that are close to each other, a co-analysis of multiple strokes is performed to match them to different nearby image lines with respect to the underlying image edges, as proposed in Ref. [8]. In this paper, we implement a similar function to distinguish temporospatially neighboring coherence strokes.

The temporal neighboring relationship is determined by the drawing order. We first take the most recent coherence stroke as the temporal neighbor of the new coherence stroke. For its spatial neighbor, we consider its parallel neighbor and 


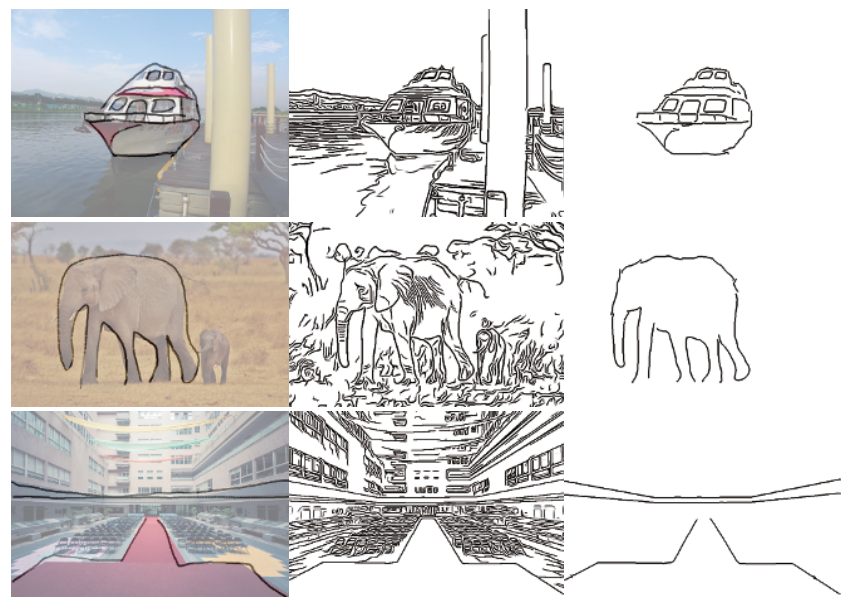

(a)

(b)

(c)

Fig. 5 Coherence line matching results. (a) Input images and user specified coherence strokes. (b) Detailed line drawings using fDoG [2]. (c) Lines extracted by coherence line matching.

its contiguous neighbor. The parallel neighbor is defined to be the neighboring stroke that is closest in distance and nearly parallel to the current stroke. They arise when the user wants to extract lines that are close to each other but find it difficult to precisely align them when using hand sketching. In order to avoid extracting the same lines when parallel neighbor strokes are given, we use an energy function to balance the results for such neighbor strokes:

$$
E_{\text {parallel }}\left(p_{\mathrm{c}}^{i}, p_{\mathrm{n}}^{j}\right)=E\left(p_{\mathrm{c}}^{i}\right)+E\left(p_{\mathrm{n}}^{j}\right)+E_{\text {conflict }}
$$

where $p_{\mathrm{c}}^{i}$ and $p_{\mathrm{n}}^{j}$ are the candidate paths derived from the current stroke $p_{\mathrm{c}}$ and the neighbor stroke $p_{\mathrm{n}}$, respectively. $E(p)$ is the energy function defined in Eq. (7). $E_{\text {conflict }}$ is an energy term designed to prevent the same lines from being extracted for parallel neighbor strokes. It is given by

$$
E_{\text {conflict }}=N_{\text {conflict }} / \max \left(N_{p_{\mathrm{c}}^{i}}, N_{p_{\mathrm{n}}^{j}}\right)
$$

where $N_{\text {conflict }}$ is the number of duplicated line segments.

The contiguous neighbor is defined to be a neighbor stroke that should be connected with the current stroke. They arise when the user wants to draw a long stroke, but, for some reason, uses two separated strokes to express this intent. In order to extract aligned, long, and coherent image lines, we use a similar energy function to that in Eq. (7) to balance the results of contiguous neighbor strokes.

$$
E_{\text {contiguous }}\left(p_{\mathrm{c}}^{i}, p_{\mathrm{n}}^{j}\right)=E\left(p_{\mathrm{c}}^{i}\right)+E\left(p_{\mathrm{n}}^{j}\right)
$$

Finally, the pair of paths $\left(p_{\mathrm{c}}^{i}, p_{\mathrm{n}}^{j}\right)$ with minimal energy is extracted. Figure 6 shows an example

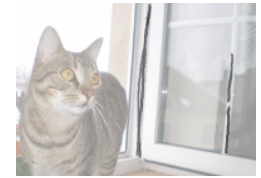

(a)

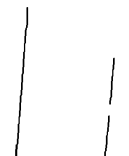

(b)

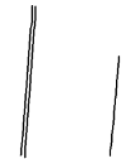

(c)
Fig. 6 Temporospatially neighboring strokes. (a) User input with parallel neighboring strokes on the left and contiguous neighboring strokes on the right. (b) Extracted lines without applying $E_{\text {parallel }}$ and $E_{\text {contiguous }}$ (c) Extracted lines using $E_{\text {parallel }}$ and $E_{\text {contiguous }}$.

where temporospatially neighboring strokes are considered or not.

\subsection{Structure line matching}

Matching of structure strokes requires us to collect evidence covered by the structure stroke. A structure cost is then used to evaluate structure similarity to the collected evidence.

Evidence collection. For a structure stroke, we need to extract line segments that have similar properties in the drawing region. The structure strokes do not need to align with the image lines, but are used for region identification. Sufficient evidence is collected as a basis to infer all other image line segments that match similar structures within the search range indicated by the user input stroke. Firstly, intersections of the user input stroke with the line segments from the line image are gathered. Secondly, for each intersection, we obtain two $3 \times 3$ patches along the tangent line on both sides of the intersected image line segment at the intersection point. Lastly, the means of these two patches are calculated. All such pairs of means are used as the evidence for testing structure similarity of line segments in the search range. Note that the search range is the same as for coherence strokes, with radius of 15 pixels. Figure 7 shows an example of evidence collection.

Structure cost. After collecting all possible evidence, the image lines that meet the search range are considered to be candidate line segments for extraction, depending on their structure similarity. For a candidate line segment $v_{i}$ having $N$ points, its color difference $D_{\text {color }}\left(v_{i}, R\right)$ to the set of evidence $R$ is defined as

$$
D_{\text {color }}\left(v_{i}, R\right)=\frac{1}{N} \sum_{m \in e_{v_{i}}} \min _{r \in R} \Delta E_{94}^{*}(m, r)
$$

where $e_{v_{i}}$ is a collection of pairs of means, calculated by the way as for the evidence, for every point of 


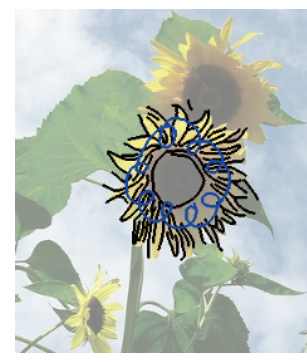

(a)

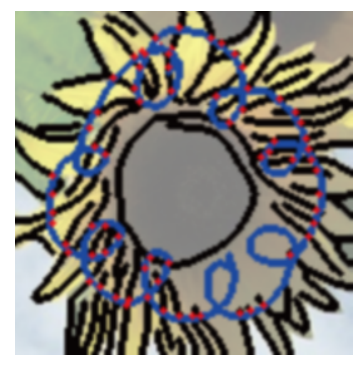

(b)

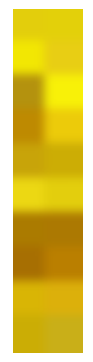

(c)
Fig. 7 Evidence collection. (a) Blue scribble: input structure stroke, black lines: candidate line segments. (b) Close-up; red dots: intersections of user strokes and candidate line segments. (c) 10 pairs of means, out of 67 in total, illustrate examples of evidence.

$v_{i}$. The operator $\Delta E_{94}^{*}$ represents the CIE94 color distance [20] measured in $L^{*} a^{*} b^{*}$ color space.

For each mean pair $m$, we find the most similar evidence pair $r$ with the smallest color difference from the evidence set $R$. The average of the smallest differences for all points of $v_{i}$ is regarded as the color difference of the entire candidate line segment. The structure cost function $C_{\text {structure }}(i)$ is then calculated for each candidate line segment $v_{i}$ :

$$
C_{\text {structure }}\left(v_{i}, R\right)=D_{\text {color }}\left(v_{i}, R\right) W\left(l_{k}\right)
$$

where $D_{\text {color }}$ is the color difference of a candidate line segment to the collected evidence set, and $l_{k}$ is the image line which line segment $v_{i}$ belongs to before line splitting. $W\left(l_{k}\right)$ is a weighting function depending on the length of the specific candidate line segment $v_{i}$, and is defined by

$$
W\left(l_{k}\right)=\frac{3}{2}-\frac{1}{2} \frac{\text { length }\left(l_{k}\right)-\operatorname{length}\left(l_{\min }\right)}{\text { length }\left(l_{\max }\right)-\operatorname{length}\left(l_{\min }\right)}
$$

where $l_{\min }$ and $l_{\max }$ are respectively the shortest and longest lengths of candidate lines before being split. This formulation causes matching to favor image lines that are longer in order to provide better line coherence.

To extract appropriate line segments, the candidate line segments are sorted by cost, ones with lower cost being the preferred ones to be extracted. The default proportion of extracted candidate line segments is $70 \%$. The user can also define the proportion of candidate line segments to be extracted. Figure 8 shows some structure line matching results with different proportions of line segments. Here, we enrich the rendering of these line drawings with colors sampled from original images to help clarify the changes between the different cases.

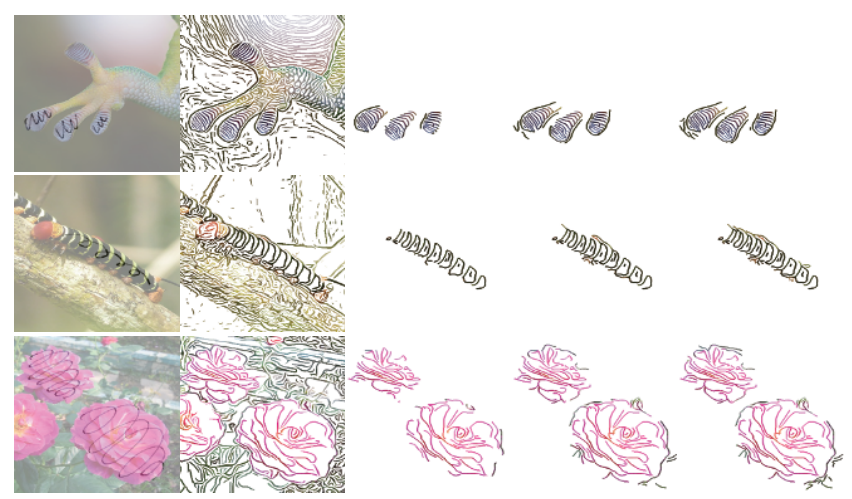

(a)

(b)

(c)

(d)

(e)

Fig. 8 Structure line matching with different proportions of line segments. (a) Input image and structure strokes. (b) Detailed line drawings using fDoG [2]. (c)-(e) Line segments with costs less than one, two, or three standard deviations respectively.

\section{Results and evaluation}

We have tested our system on a wide variety of images across different users and generated 14 line abstractions with only a small number of user strokes. A few examples can be found in Fig. 9 and we refer the reader to the Electronic Supplementary Material for a full gallery.

\subsection{Evaluation}

In this section, we give the results of several experiments to evaluate the performance of our system against naive and state-of-the-art methods. In particular, our system is compared with two stateof-the-art methods by Yang et al. [16] and Su et al. [8] (EZ-Sketching), which share the same goal as our system of generating long coherent lines from user strokes. We also implemented two naive approaches for a baseline comparison, including: (i) extracting lines that are near to the user strokes, within a distance threshold of 15 pixels (NN); and (ii) using all the lines that intersect user strokes (NI).

Performance of coherence line matching. We evaluate the performance of our coherence line matching algorithm against above four alternatives in terms of visual quality and edge detection accuracy with respect to the ground truth. To do so, we used the same benchmark as Yang et al. [16] and took $\mathrm{gPb}[6]$ edge maps as input reference line images to our system. For a fair comparison, we imitated 10 results shown in Ref. [16] by carefully tracing their user strokes using our coherence strokes. These coherence strokes were also used as input to EZ- 
Sketching [8] to generate the outputs for comparison. Figure 10 shows a side-by-side comparison of the results. Our results are visually comparable to those of EZ-Sketching [8], and superior to those of Ref. [16] and both naive approaches in terms of smoothness and conciseness. We further used the precision $P$,
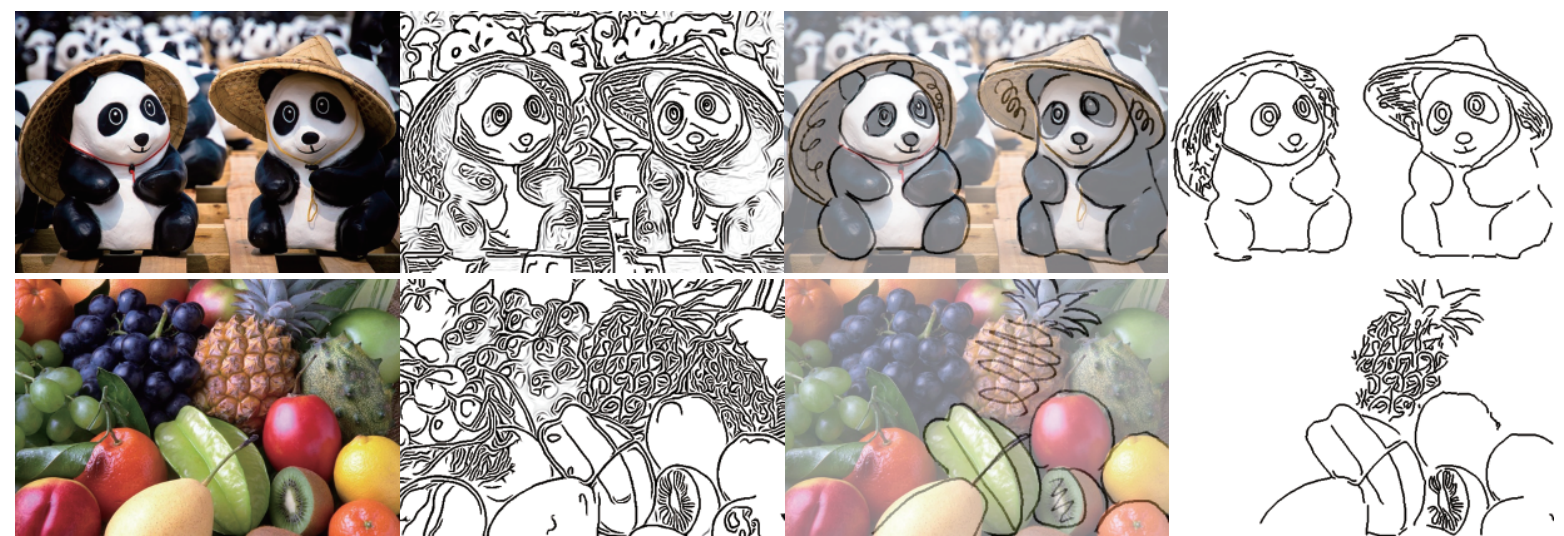

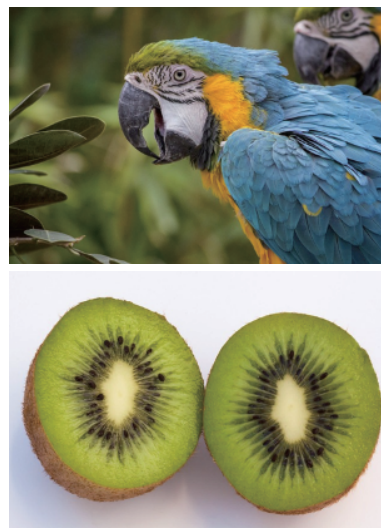

(a)

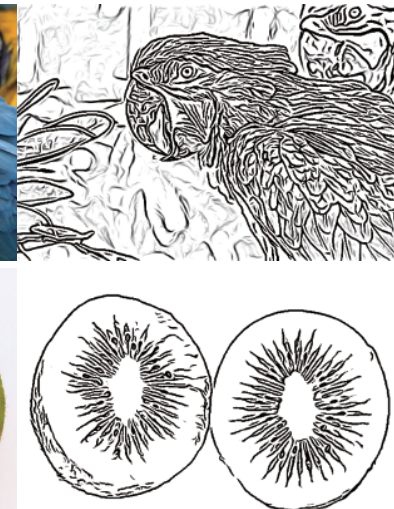

(b)
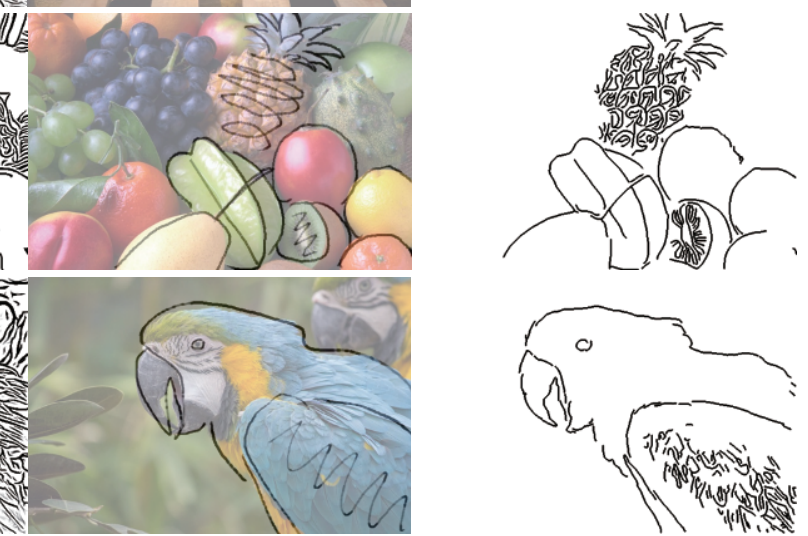

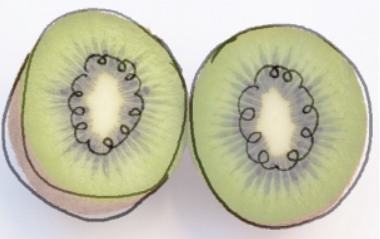

(c)

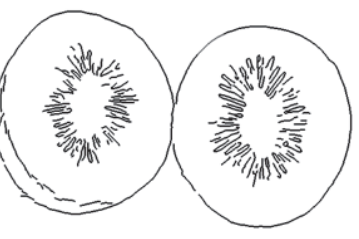

(d)

Fig. 9 Four results generated using our system. (a) Input image. (b) Detailed line drawings by fDoG [2]. (c) User strokes. (d) Final line abstractions.

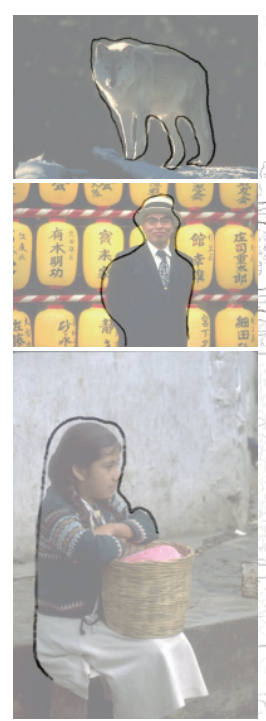

(a)

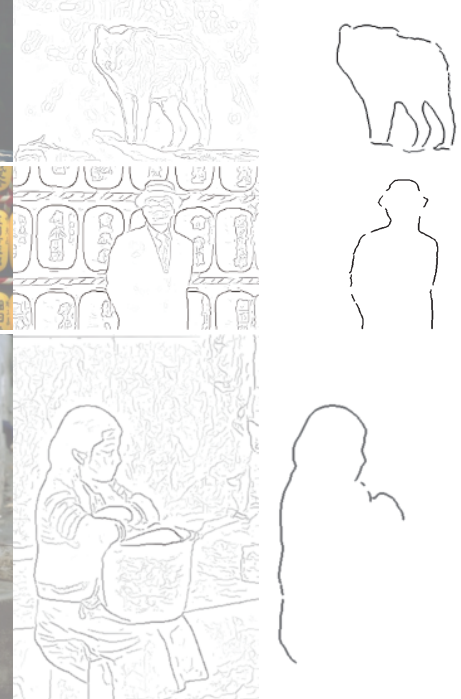

(b) (c)
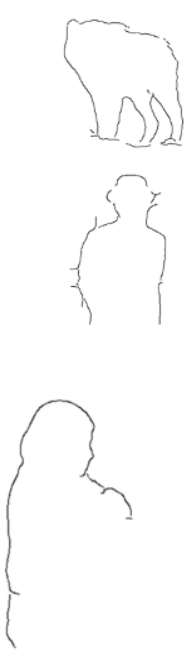

(d)<smiles>C1=CCCCCC1</smiles><smiles>C1CCCCCCCCCCC1</smiles><smiles>C1CCCCCCC1</smiles>

(e)<smiles>c1ccc(CSc2ccccn2)cc1</smiles>

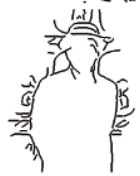<smiles>CCCCC(CCC)(CCCC)C(C)(C)C</smiles>

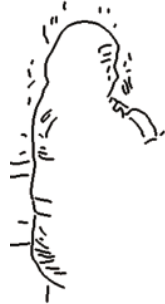

(f)

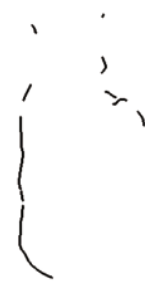

(g)

Fig. 10 Comparison with four other methods. (a) Input image and user strokes. (b) Ground truths corresponding to detailed line drawings by gPb [6]. (c)-(g) Lines extracted by (c) our system, (d) Yang et al. [16], (e) EZ-Sketching [8], (f) naive near neighbor search (NN), and (g) naive line-stroke intersection test (NI). 
recall $R$, and $F$-measure (the weighted harmonic mean of $P$ and $R$ ) to evaluate edge detection accuracy. Table 1 shows that our algorithm achieves comparable performance to Ref. [16] and clearly outperforms EZ-Sketching [8] in terms of $F$-measure. Note that Yang et al.s method achieves better recall than ours as the ground truths often contain lines that are not expected by the user. For example, in the second row of Fig. 10(d), the noisy branches around the man's contour come from the shape of the lanterns in the background, which are also included in the ground truth (see Fig. 10(b)). On the other hand, although EZ-Sketching snapped user strokes to nearby edges, it tended to retain the style of the user strokes instead of emphasizing the precision of the refined strokes. Therefore, the precision and recall are relatively low.

Performance of structure line matching. Since neither Yang et al.'s system nor EZ-Sketching are designed to handle scribbles, we evaluated the performance of the structure line matching algorithm

Table 1 Edge detection accuracy

\begin{tabular}{lccc}
\hline Method & $F$-measure & Recall & Precision \\
\hline Ours & 0.539 & 0.388 & 0.972 \\
Yang et al. [16] & 0.549 & 0.402 & 0.939 \\
EZ-Sketching [8] & 0.398 & 0.279 & 0.766 \\
\hline NI & 0.359 & 0.226 & 0.918 \\
NN & 0.608 & 0.538 & 0.733 \\
\hline
\end{tabular}

(a)

(b)

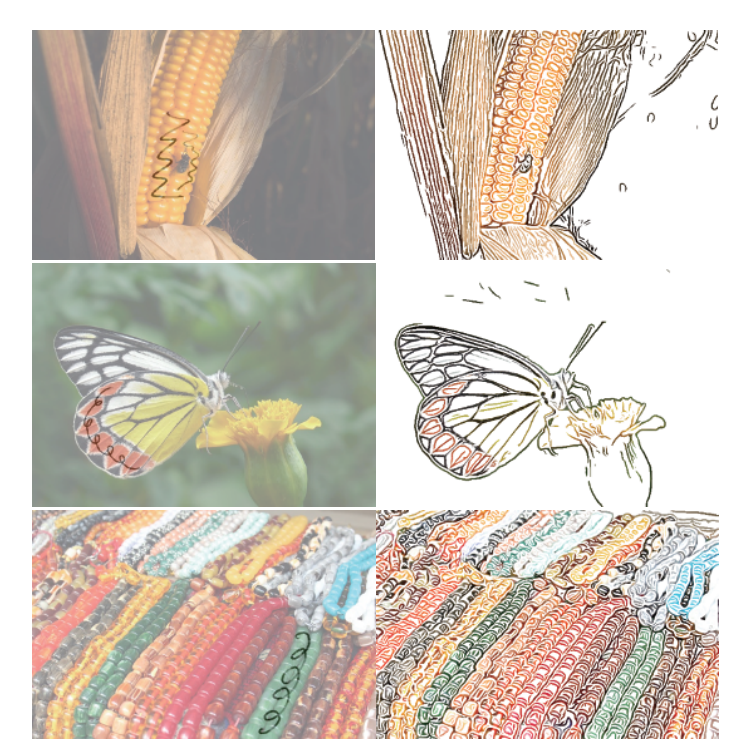

only in comparison with naive methods ( $\mathrm{NN}$ and NI). A side-by-side comparison can be found in Fig. 11. Note that we enrich the rendering of line drawings with colors sampled from the original images to better show how our algorithm can effectively capture lines with similar features, while the naive approaches tend to generate results with excessive (NN) or insufficient (NI) details.

User study. We evaluated the overall quality of line abstractions by conducting a user study. Specifically, we prepared two sets of images, each of which contains 10 example images with predrawn user strokes. One set was used to evaluate coherence line matching while the other was used for structure line matching. For both sets, we generated 3 results for each example using our system and two naive methods (NN and NI). The result generated by EZ-Sketching [8] was also included for the set used to evaluate coherence line matching. During each trial, the subject was shown the original image with user strokes and line abstractions by different methods. The subject was then asked to grade each result using a score of $1-5$ (the higher, the better) according to the degree of completeness, cleanness, and expectation as comparing to the input strokes. The average score over 11 subjects is given in Fig. 12 .

For coherence line matching, there was a statistically significant difference between groups as determined by one-way ANOVA $(F(3,36)=21.646$,
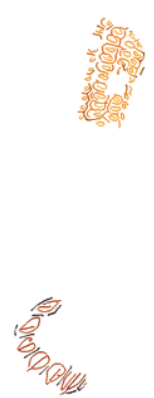

(c)
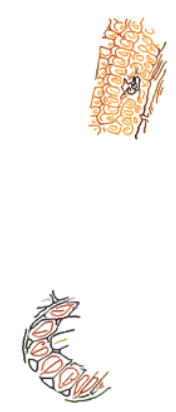

(d)

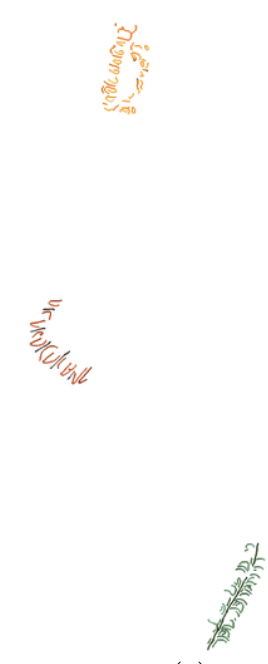

(e)

Fig. 11 Comparison with naive methods. (a) Input image and user strokes. (b) Detailed color line drawings by fDoG [2]. (c)-(e) Lines extracted by (c) our system with costs less than one standard deviation, (d) naive near neighbor search (NN), and (e) naive line-stroke intersection test (NI). 


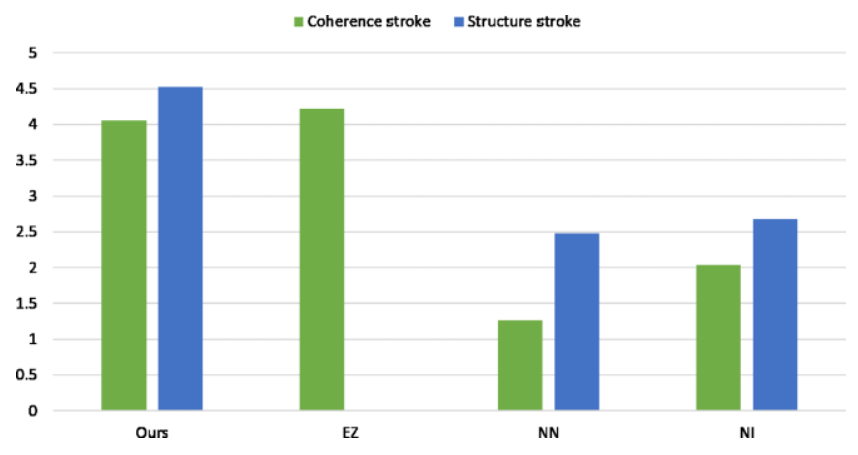

Fig. 12 Average scores of different methods in the user study (the higher, the better).

$p<0.001)$. An LSD post-hoc test revealed that the score for NN $(1.26 \pm 0.2, p<0.001)$ and NI $(2.04 \pm 0.26, p<0.001)$ was statistically significantly lower than that for our method $(4.05 \pm 0.28)$. There was no statistically significant difference between our method and EZ-Sketching [8] $(p=0.152)$. According to the participant feedback, some of them cared more about smoothness and completeness of the coherence lines rather than their precision. Since EZSketching [8] refined the user strokes to snap them to nearby edges, while our method extracted lines from images which were originally composed of many incoherent line segments, EZ-Sketching [8] tended to get higher scores for some participants.

For structure line matching, there was a statistically significant difference between groups as determined by one-way ANOVA $(F(2,27)=$ $56.429, p<0.001)$. An LSD post-hoc test revealed that the score of NN $(2.48 \pm 0.62, p<0.001)$ and NI $(2.68 \pm 0.53, p<0.001)$ was statistically significantly lower than that for our method $(4.53 \pm 0.14)$. There was no statistically significant difference between $\mathrm{NN}$ and NI $(p=0.355)$.

System usability. We conducted a small user study with 3 subjects to test the usability of our system against EZ-Sketching [8]. During each trial, the subject was asked to generate a line drawing with a comparable level of details to a given reference image using our system and EZ-Sketching [8], and we recorded how long the subjects took to finish the line drawings. The timing statistics can be found in Table 2, and examples are shown in Fig. 13. The results indicate that users take more time when using EZ-Sketching [8] to generate a line drawing with a target level of detail.

Speed. Once the user draws a stroke, our system
Table 2 Time taken to generate line drawings

\begin{tabular}{lcc}
\hline & Our method & EZ-Sketching \\
\hline Subject \#1 & $144 \mathrm{~s}$ & $192 \mathrm{~s}$ \\
Subject \#2 & $79 \mathrm{~s}$ & $158 \mathrm{~s}$ \\
Subject \#3 & $39 \mathrm{~s}$ & $100 \mathrm{~s}$ \\
\hline Average time & $87 \mathrm{~s}$ & $150 \mathrm{~s}$ \\
\hline
\end{tabular}
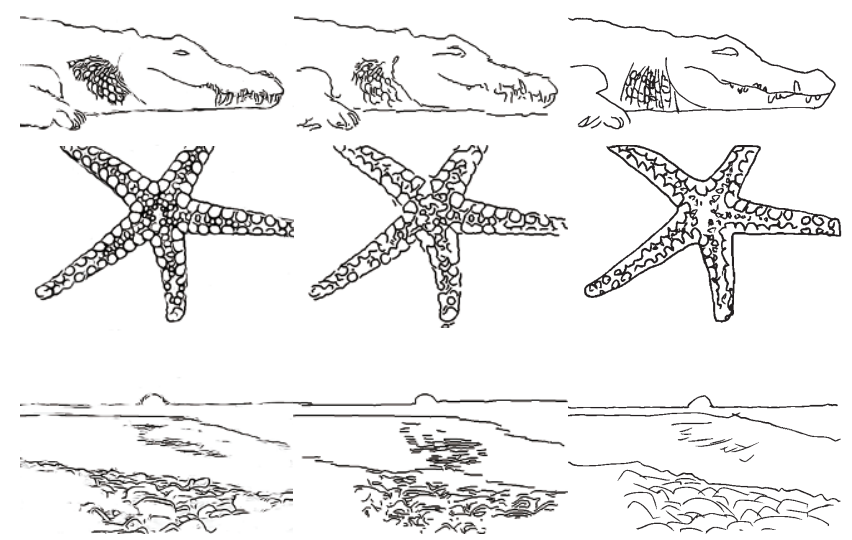

(a)

(b)

(c)

Fig. 13 Results generated by different systems. (a) Reference line drawings. (b) Line drawing generated by our system. (c) Line drawing generated by EZ-Sketching [8].

can extract the corresponding line segments at an interactive rate. For all the images we tested, our system took on average less than one second to perform coherence line matching or structure line matching. The timing complexity of both line matching algorithms is proportional to the number of candidate line segments involved in the computation.

\subsection{Limitations}

The quality of the extracted lines is currently limited by the input detailed line drawings. First, our system can not extract lines that are not present in the dataset. For instance, the duckling shown in Fig. 14(a) presents a jagged outline, as a result of which most image filtering algorithms fail to generate long coherent lines (see Fig. 14(b)). In such cases, our system can not extract long coherent lines using coherence strokes (see Fig. 14(c)). On the other hand, the quality of the extracted structure lines depends on the degree of color diversity in the input image. Since structure line matching depends on color differences of line segments, the system may fail to extract meaningful structure lines if the reference image lacks color diversity with the stroke's ROI (see Figs. 14(d)-14(f)). 


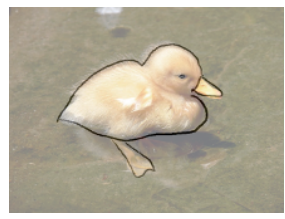

(a)

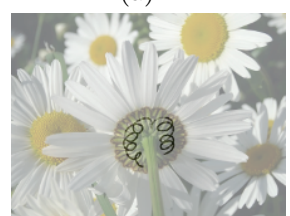

(d)

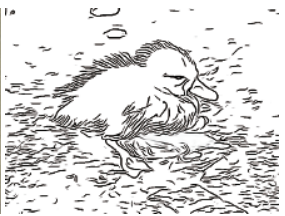

(b)

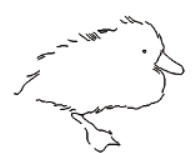

(c)

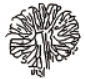

(f)
Fig. 14 Limitations. (a, d) Input images with coherence strokes and structure strokes respectively. (b, e) Detailed line drawings of (a, d) respectively. (c, f) Line abstractions produced by our system. Note that (c) fails to extract coherence lines due to the noisy line segments along the duck's boundary in (b). Due to the small color difference between sepals and flower stem in (d), our system extracts lines from both sepals and stem even though the user is only interested in the sepal region.

\section{Conclusions}

In this work, we have presented a novel interactive system for generating a concise, semantic line abstraction guided by a few user strokes. The user strokes are classified into coherence strokes and structure strokes to facilitate extracting effective line drawings from arbitrary images. For a coherence stroke, we build a graph and apply an energy function to extract lines that are coherent and aligned with user strokes. For a structure stroke, we calculate the color difference between the candidate lines and the evidence, and allow lines with similar structures to be extracted. Our system is efficient and can respond in real time. Its effectivity has been verified by comparing it with other line extraction approaches. The results show that our approach is superior to other systems in terms of quality and efficiency.

\section{Acknowledgements}

We are grateful to the anonymous reviewers for their comments and suggestions. The work was supported in part by the "Ministry of Science and Technology of Taiwan" (Nos. 103-2221-E-007-065-MY3 and 105-2221-E-007-104-MY2).

Electronic Supplementary Material Supplementary material is available in the online version of this article at http://dx.doi.org/10.1007/s41095-016-0076-y.

\section{References}

[1] Canny, J. A computational approach to edge detection. IEEE Transactions on Pattern Analysis and Machine Intelligence Vol. PAMI-8, No. 6, 679-698, 1986.

[2] Kyprianidis, J. E.; Döllner, J. Image abstraction by structure adaptive filtering. In: Proceedings of the EG UK Theory and Practice of Computer Graphics, 5158, 2008.

[3] Winnemöeller, H.; Olsen, S. C.; Gooch, B. Real-time video abstraction. ACM Transactions on Graphics Vol. 25, No. 3, 1221-1226, 2006.

[4] Winnemöller, H.; Kyprianidis, J. E.; Olsen, S. C. XDoG: An extended difference-of-Gaussians compendium including advanced image stylization. Computers \&s Graphics Vol. 36, No. 6, 740-753, 2012.

[5] Arbelaez, P.; Maire, M.; Fowlkes, C.; Malik, J. Contour detection and hierarchical image segmentation. IEEE Transactions on Pattern Analysis and Machine Intelligence Vol. 33, No. 5, 898-916, 2011.

[6] Maire, M.; Arbelaez, P.; Fowlkes, C.; Malik, J. Using contours to detect and localize junctions in natural images. In: Proceedings of the IEEE Conference on Computer Vision and Pattern Recognition, 1-8, 2008.

[7] Limpaecher, A.; Feltman, N.; Treuille, A.; Cohen, M. Real-time drawing assistance through crowdsourcing. ACM Transactions on Graphics Vol. 32, No. 4, Article No. 54, 2013.

[8] Su, Q.; Li, W. H. A.; Wang, J.; Fu, H. EZsketching: Three-level optimization for error-tolerant image tracing. ACM Transactions on Graphics Vol. 33, No. 4, Article No. 54, 2014.

[9] Gleicher, M. Image snapping. In: Proceedings of the 22nd Annual Conference on Computer Graphics and Interactive Techniques, 183-190, 1995.

[10] Li, Y.; Sun, J.; Tang, C.-K.; Shum, H.-Y. Lazy snapping. ACM Transactions on Graphics Vol. 23, No. 3, 303-308, 2004.

[11] Lee, Y. J.; Zitnick, C. L.; Cohen, M. F. ShadowDraw: Real-time user guidance for freehand drawing. ACM Transactions on Graphics Vol. 30, No. 4, Article No. 27, 2011.

[12] Iarussi, E.; Bousseau, A.; Tsandilas, T. The drawing assistant: Automated drawing guidance and feedback from photographs. In: Proceedings of the ACM Symposium on User Interface Software and Technology, 2013.

[13] Dixon, D.; Prasad, M.; Hammond, T. iCanDraw: Using sketch recognition and corrective feedback to assist a user in drawing human faces. In: Proceedings of the SIGCHI Conference on Human Factors in Computing Systems, 897-906, 2010.

[14] Lu, J.; Barnes, C.; DiVerdi, S.; Finkelstein, A. RealBrush: Painting with examples of physical media. ACM Transactions on Graphics Vol. 32, No. 4, Article 
No. 117, 2013.

[15] Berger, I.; Shamir, A.; Mahler, M.; Carter, E.; Hodgins, J. Style and abstraction in portrait sketching. ACM Transactions on Graphics Vol. 32, No. 4, Article No. 55, 2013.

[16] Yang, S.; Wang, J.; Shapiro, L. Supervised semantic gradient extraction using linear-time optimization. In: Proceedings of the IEEE Conference on Computer Vision and Pattern Recognition, 2826-2833, 2013.

[17] Wobbrock, J. O.; Wilson, A. D.; Li, Y. Gestures without libraries, toolkits or training: A $\$ 1$ recognizer for user interface prototypes. In: Proceedings of the 20th Annual ACM Symposium on User Interface Software and Technology, 159-168, 2007.

[18] Orbay, G.; Kara, L. B. Beautification of design sketches using trainable stroke clustering and curve fitting. IEEE Transactions on Visualization and Computer Graphics Vol. 17, No. 5, 694-708, 2011.

[19] Floyd, R. W. Algorithm 97: Shortest path. Communications of the ACM Vol. 5, No. 6, 345, 1962.

[20] McDonald, R.; Smith, K. J. CIE94-A new colourdifference formula. Journal of the Society of Dyers and Colourists Vol. 111, No. 12, 376-379, 1995.

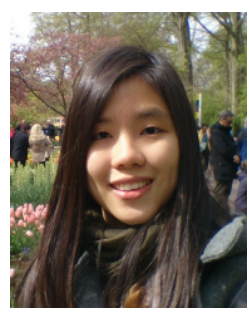

Hui-Chi Tsai received her bachelor and master degrees in computer science from "National Tsing Hua University", Taiwan, China. She is currently a software engineer in the Information and Communications Research Laboratories, Industrial Technology Research Institute, Taiwan, China. Her research interests include computer graphics and computer vision.

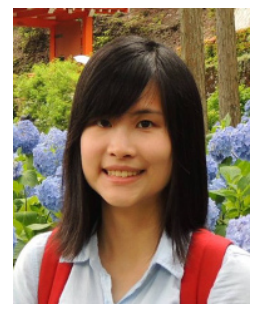

Ya-Hsuan Lee received her B.S. degree in computer science from "National Tsing Hua University", Taiwan, China, in 2016. She is currently working at MediaTek as an engineer. Her research interests include computer graphics and computer vision.

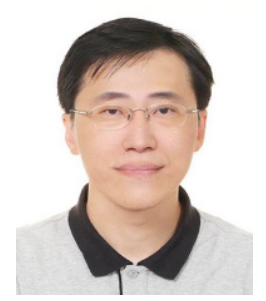

Ruen-Rone Lee received his Ph.D. degree in computer science from "National Tsing Hua University", Taiwan, China, in 1994. From 1994 to 2010, he worked in several IC design companies for graphics hardware and software development. Later, from 2010 to 2015, he was an associate researcher with the Department of Computer Science, "National Tsing Hua University". He is currently a deputy director in the Information and Communications Research Laboratories, Industrial Technology Research Institute, Taiwan, China. His research interests include computer graphics, non-photorealistic rendering, and graphics hardware architecture design. He is a member of the IEEE Computer Society and the ACM SIGGRAPH.

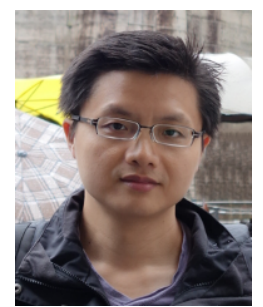

Hung-Kuo Chu received his Ph.D. degree from the Department of Computer Science and Information Engineering, "National Cheng Kung University", Taiwan, China, in 2010. He is currently an associate professor at the Department of Computer Science, "National Tsing Hua University". His research interests focus on shape understanding, smart manipulation, perception-based rendering, recreational graphics, and human computer interaction.

Open Access The articles published in this journal are distributed under the terms of the Creative Commons Attribution 4.0 International License (http:// creativecommons.org/licenses/by/4.0/), which permits unrestricted use, distribution, and reproduction in any medium, provided you give appropriate credit to the original author(s) and the source, provide a link to the Creative Commons license, and indicate if changes were made.

Other papers from this open access journal are available free of charge from http://www.springer.com/journal/41095. To submit a manuscript, please go to https://www. editorialmanager.com/cvmj. 